\title{
Migration process of very low-frequency events based on a chain-reaction model and its application to the detection of preseismic slip for megathrust earthquakes
}

\author{
Keisuke Ariyoshi ${ }^{1}$, Toru Matsuzawa ${ }^{2}$, Jean-Paul Ampuero ${ }^{3}$, Ryoko Nakata ${ }^{1}$, Takane Hori ${ }^{1}$, \\ Yoshiyuki Kaneda $^{1}$, Ryota Hino ${ }^{2}$, and Akira Hasegawa ${ }^{2}$ \\ ${ }^{1}$ Earthquake and Tsunami Research Project for Disaster Prevention, Japan Agency for Marine-Earth Science and Technology, \\ Yokohama 236-0001, Japan \\ ${ }^{2}$ Research Center for Prediction of Earthquakes and Volcanic Eruptions, Graduate School of Science, \\ Tohoku University, Sendai 980-8578, Japan \\ ${ }^{3}$ Seismological Laboratory, California Institute of Technology, Pasadena, California 91125-2100, USA
}

(Received March 29, 2010; Revised September 29, 2010; Accepted September 30, 2010; Online published August 27, 2012)

\begin{abstract}
In order to reproduce slow earthquakes with short duration such as very low frequency events (VLFs) migrating along the trench direction as swarms, we apply a 3-D subduction plate boundary model based on the slowness law of rate- and state-dependent friction, introducing close-set numerous small asperities (rate-weakening regions) at a depth of $30 \mathrm{~km}$ under high pore pressure condition, in addition to a large asperity. Our simulation indicates that swarms of slip events occur repeatedly at the small asperities, and these events are similar to the observed slow earthquake group, especially to VLF, on the basis of the relation between characteristic duration and seismic moment. No slip events occur there without the small asperities, which mean that the close-set numerous small asperities may be one of the necessary conditions for generating the short-duration slow earthquakes such as VLFs. In the preseismic stage of the megathrust earthquakes that occur at the large asperity, the swarms of VLFs have higher migration speeds and higher moment release rate as well as shorter recurrence interval. Thus, monitoring the migration of slow earthquakes may be useful in imaging the preseismic slip of megathrust earthquakes.
\end{abstract}

Key words: Numerical simulation, friction law, slow earthquake, low-frequency tremor, preseismic change.

\section{Introduction}

By virtue of dense networks of GPS and highly sensitive seismic stations, "slow earthquakes" (Ide et al., 2007), including slow slip events (SSEs), deep low-frequency tremors (LFTs) and very low-frequency events (VLFs), have been detected worldwide in the deep portions of subduction plate boundaries (e.g., Obara, 2002; Rogers and Dragert, 2003; Schwartz and Rokosky, 2007). Most of the slow earthquakes, including VLF swarms (Obara and Sekine, 2009), migrate at rates of 2-20 km/day (Schwartz and Rokosky, 2007) along the trench. They are thought to be located on the subduction plate boundary at a depth of $30 \mathrm{~km}$ (e.g., Shelly et al., 2006), in the transition zone between the stably sliding regions and the deeper outskirts of large asperities that generate megathrust earthquakes (Dragert et al., 2007). Since some LFTs are found to be modulated by Earth tides (Nakata et al., 2008; Rubinstein et al., 2008) and the moment release rate of the slow earthquake group is much smaller than that of regular earthquakes (Ide et al., 2007), they likely have low-stress drop and are sensitive to shear stress perturbations possibly in-

Copyright (C) The Society of Geomagnetism and Earth, Planetary and Space Sciences (SGEPSS); The Seismological Society of Japan; The Volcanological Society of Japan; The Geodetic Society of Japan; The Japanese Society for Planetary Sciences; TERRAPUB.

doi:10.5047/eps.2010.09.003 duced by the preseismic slip of nearby megathrust earthquakes.

Recently, numerical simulations that use rate- and statedependent friction laws (RSF) (Dieterich, 1979; Ruina, 1983) have explained the SSE migration process. Shibazaki and Shimamoto (2007) introduced a friction law developed from the RSF and succeeded in reproducing the SSE migration at a speed of approximately $10 \mathrm{~km} /$ day. However, they did not discuss possible changes in the SSE migration characteristics during the preseismic stage of megathrust earthquakes. Liu and Rice (2005) pointed out the relationship between the spatio-temporal distribution of the SSE and nucleation zones of thrust earthquakes, which would be useful for estimating the location of the next megathrust earthquake, but Liu and Rice (2005) and Shibazaki and Shimamoto (2007) did not reproduce swarms of the shortduration slow earthquakes composed of LFT and/or VLF.

In the present study, we perform numerical simulations of a three-dimensional subduction plate boundary, and reproduce slow earthquake swarms mainly composed of VLF by introducing close-set numerous small asperities (rateweakening regions) with high spatio-temporal resolution. We also propose a new method to detect precursory change around the large asperity prior to a megathrust earthquake, focusing on the spatio-temporal change of migration speed and moment release rate for nearby slow earthquakes. 

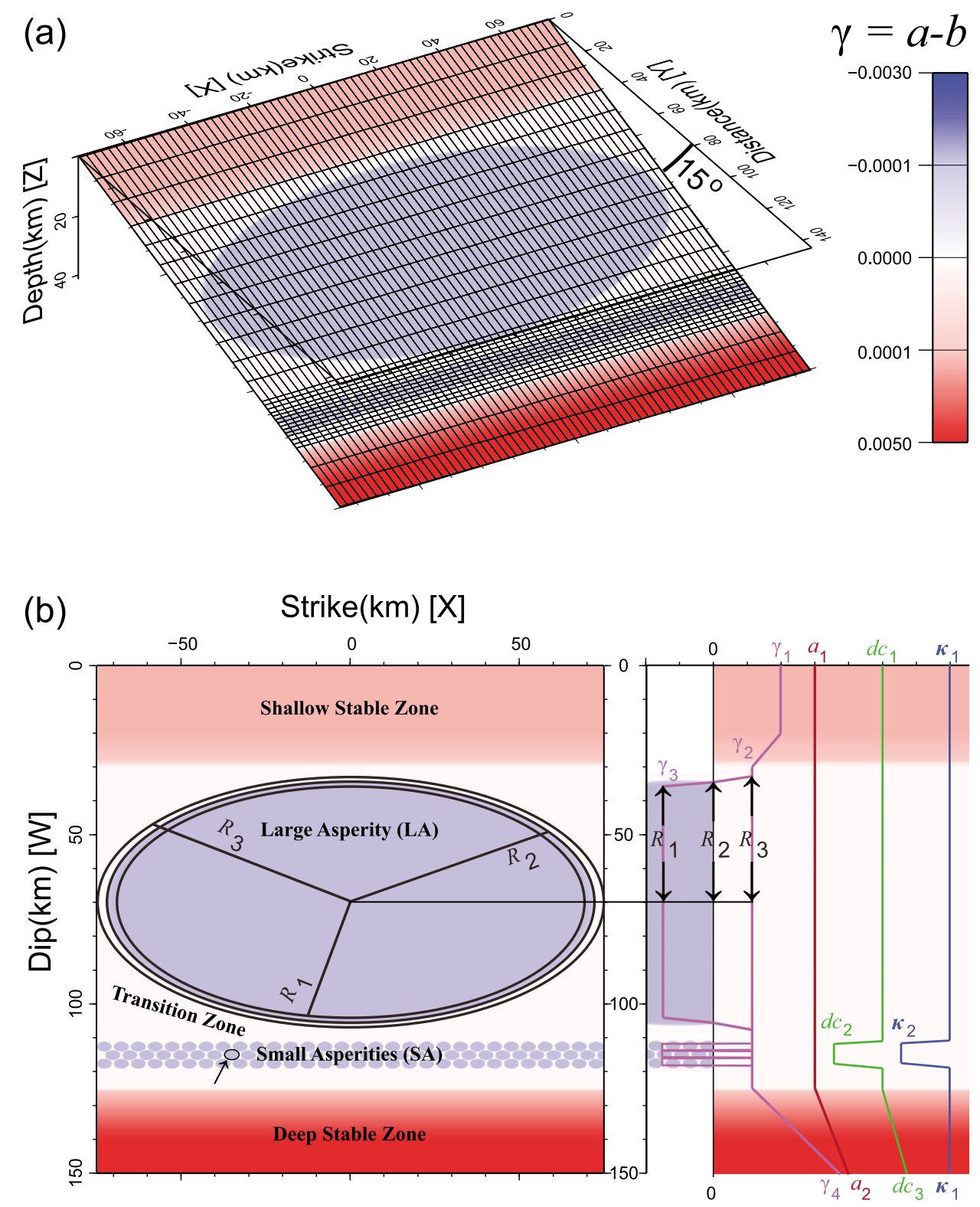

Fig. 1. (a) Schematic view of the 3-D simulation model of a plate boundary with frictional parameter $\gamma=a-b$. Note that the actual cell size in the simulation is more than eight times smaller than shown here. (b) Frictional parameters $\left(a, \gamma, d_{\mathrm{c}}, \kappa\right.$ (see Eq. (2)) as functions of distance along the dip direction from the surface, where $\left(a_{1}, a_{2}\right)=(2,5)\left[\times 10^{-3}\right],\left(\gamma_{1}, \gamma_{2}, \gamma_{3}, \gamma_{4}\right)=(0.5,0.01,-0.3,4.9)\left[\times 10^{-3}\right],\left(d_{\mathrm{c} 1}, d_{\mathrm{c} 2}, d_{\mathrm{c} 3}\right)=(10,0.43,100)$ $[\mathrm{mm}]$, and $\left(\kappa_{1}, \kappa_{2}\right)=(1.0,0.1)$. Half the length of the minor axis (along dip) of the elliptical asperity takes the following values: for LA, $\left(R_{1}, R_{2}, R_{3}\right)=(35,36.25,37.5)[\mathrm{km}]$ and for SA, $\left(r_{1}, r_{2}, r_{3}\right)=(1.33,1.5,1.67)[\mathrm{km}]$, where the aspect ratios for LA and SA are 2.0 and 1.5 , respectively. The distance between central points of SA along strike and dip direction is 2 and $2.5 \mathrm{~km}$, respectively. The time histories for SA, indicated by the arrow, are shown in Fig. 2.

\section{Simulation Model}

The model consists of a planar plate interface dipping at 15 degrees from the free surface in a homogeneous elastic half-space (Fig. 1(a)) with a periodic boundary condition along the strike direction. The plate interface is divided into 1,024 (strike) $\times 293$ (dip) cells.

Slip is assumed to occur in the pure dip direction and to obey the quasi-static equilibrium between shear and frictional stresses by introducing a radiation damping term (Rice, 1993):

$$
\mu_{i} \sigma_{i}=\sum_{j=1}^{N} K_{i j}\left(u_{j}(t)-V_{\mathrm{pl}} t\right)-\frac{G}{2 \beta} \frac{d u_{i}}{d t},
$$

Here, the subscripts $i$ and $j$ denote the location indices of a receiver and a source cell, respectively. The left hand side of Eq. (1) describes frictional stress, where $\mu$ and $\sigma$ is friction coefficient and effective normal stress, respectively. The right hand side describes the shear stress in the $i$-th cell caused by dislocations, where $K_{i j}$ is the Green's function for the shear stress (Okada, 1992) on the $i$-th cell, $N$ is the total number of cells, $V_{\mathrm{pl}}$ is the relative speed of the two plates, $t$ denotes time, $G$ is rigidity, $\beta$ is the shear wave speed. $K_{i j}$ is calculated from the quasi-static solution for uniform pure dip-slip $u$ relative to average slip $V_{\mathrm{pl}} t$ (Savage, 1983) over a rectangular dislocation in the $j$-th cell. Parts of the first term of the right-hand side are written as convolutions, by exploiting the along-strike invariance of 
the Green's function, and efficiently computed by the Fast Fourier Transform (e.g., Rice, 1993; Liu and Rice, 2005).

In Eq. (1), the effective normal stress $\sigma$ is given by

$$
\sigma_{i}(z)=\kappa(z)\left(\rho_{\text {rock }}-\rho_{\mathrm{w}}\right) g z,
$$

where $\rho_{\text {rock }}$ and $\rho_{\mathrm{w}}$ are the densities of rock and water, respectively, $g$ is the acceleration due to gravity, and $z$ is the depth. The function $\kappa(z)$ is a super-hydrostatic pore pressure factor, as given in Fig. 1(b). We assume that a high-pore-pressure system locally exists around a depth of $30 \mathrm{~km}$ based on the high- $V_{p} / V_{s}$ zones in southwestern Japan (Shelly et al., 2006; Matsubara et al., 2008). The increase in pore pressure is probably due to the dehydration derived from the change in facies in the slab (e.g., Hacker $e t$ al., 2003). Ariyoshi et al. (2007a) estimated that the value of $\kappa$ is 0.1 for the deeper part (>30 km depth) based on the post-seismic slip propagation speed. On the basis of the stress field observation in northeastern and southwestern Honshu, Japan, Wang and Suyehiro (1999) suggested that the apparent frictional coefficient is approximately 0.03 , which is consistent with $\kappa=0.1$.

The friction coefficient $\mu$ is assumed to obey an RSF law (Dieterich, 1979; Ruina, 1983), as given by

$$
\begin{aligned}
& \mu=\mu_{0}+a \log \left(V / V_{0}\right)+b \log \left(V_{0} \theta / d_{\mathrm{c}}\right), \\
& d \theta / d t=1-V \theta / d_{\mathrm{c}},
\end{aligned}
$$

where $a$ and $b$ are friction coefficient parameters, $d_{\mathrm{c}}$ is the characteristic slip distance associated with $b, \theta$ is a state variable for the plate interface, $V$ is the slip velocity, and $\mu_{0}$ is a reference friction coefficient defined at a constant reference slip velocity of $V_{0}$.

We consider a model with close-set numerous small asperities on the deeper outskirt of a great asperity, as proposed by Dragert et al. (2007). In the present study, an asperity denotes a region with $a-b=\gamma<0$, following Boatwright and Cocco (1996). The plate interface is demarcated into five parts, as shown in Fig. 1(b): (i) one large asperity (LA), (ii) 90 small asperities (SAs), (iii) a shallow stable zone, (iv) a deep stable zone, and (v) a transition zone $(\gamma \sim+0)$. The values of frictional parameters as described in the caption of Fig. 1(b) are based on rock laboratory results (e.g., Blanpied et al., 1998), which will be discussed later.

The constant parameters in the present study are $V_{\mathrm{pl}}=$ $4.0 \times 10^{-2} \mathrm{~m} / \mathrm{yr}$ (or $\left.1.3 \times 10^{-9} \mathrm{~m} / \mathrm{s}\right), G=30 \mathrm{GPa}, \beta=$ $3.75 \mathrm{~km} / \mathrm{s}, \rho_{\text {rock }}=2.75 \times 10^{3} \mathrm{~kg} / \mathrm{m}^{3}, \rho_{\mathrm{w}}=1.0 \times 10^{3} \mathrm{~kg} / \mathrm{m}^{3}$, $g=9.8 \mathrm{~m} / \mathrm{s}^{2}, V_{0}=1 \mu \mathrm{m} / \mathrm{s}, \mu_{0}=0.6$, and Poisson's ratio $\varepsilon=0.25$.

All cells are smaller than characteristic length scale $L_{\mathrm{b}}=$ $G d_{\mathrm{c}} / \sigma b$ (Rubin and Ampuero, 2005), which is related to the minimum size of nucleation zones and to the characteristic size of the process zone of propagating transients, including in the frictionally stable region $(\gamma>0)$. For all of the small asperities, the cell size (strike, dip) is ( $150 \mathrm{~km} / 1,024$ cells, $10 \mathrm{~km} / 74$ cells), which is (nearly $1 / 4$ times) smaller than $L_{\mathrm{b}}(0.57 \mathrm{~km})$. We uniformly set the initial shear stress at the steady state friction value at a rate of $0.9 V_{\mathrm{pl}}$. The validity of the cell size and initial conditions will also be discussed.

\section{Characteristics of Slip Events in LA and SA Based on the Moment Release Amounts}

Figures 2(a-c) show the frictional coefficient (or shear stress normalized by the effective normal stress) and the slip velocity normalized with respect to $V_{\mathrm{pl}}$ on a common-logarithmic scale averaged on LA and SA at $(X, W)=(-35,115)$, as indicated by the arrow in Fig. 1(b). Figure 2(a) shows that LA generates a periodic $\left(T_{\mathrm{r}}=116.3\right.$ years $)$ megathrust $\left(M_{\mathrm{w}}=7.9\right)$ earthquake, where seismic coupling $\left(D_{\mathrm{s}} / V_{\mathrm{pl}} T_{\mathrm{r}}\right)$ is $71 \%$ if we define the seismic $\operatorname{slip}\left(D_{\mathrm{s}}\right)$ as slip that exceeds $1 \mathrm{~cm} / \mathrm{sec}$. On the other hand, each SA causes approximately 200 non-characteristic slip events in a cycle of megathrust earthquakes but only few events include seismic slip.

The amount of stress drop of the slip events in Fig. 2 (0.02-0.08 MPa) is comparable to SSE (0.04-0.06 MPa) (Miyazaki et al., 2006). The moment release rate of the slip event averaged over the SAs calculated from $\dot{M}_{\mathrm{o}}=$ $G S V_{\max }$, where $V_{\max }$ is the maximum of the slip velocity averaged over the SAs and $S$ is the area for one $\mathrm{SA}(\gamma<0)$, is as small as $10^{-6}$ to $10^{-7}$ times that of the megathrust earthquake in LA.

Duration time $(\Delta t)$ of slip events for LA is defined as a condition of maximum slip velocity in LA higher than $1 \mathrm{~cm} / \mathrm{sec}$, which gives the duration time several minutes. For SA, Figs. 2(b) and 2(c) shows that slip events occur with wide range of slip velocity due to interaction. In this study, we define the duration time on the basis of moment release acceleration $\left(\ddot{M}_{\mathrm{o}}\right)$ instead of slip velocity. Figure $2(\mathrm{~d})$ shows the time history of the moment release rate for a slip event. Since the moment release acceleration should be temporally smaller around the peak of moment release rate, we define the duration time for SA as $\left|\ddot{M}_{\mathrm{o}}(t)\right|<1 / 2 \mathrm{~min}$ $\left(\left|\ddot{M}_{\text {omin }}\right|,\left|\ddot{M}_{\text {omax }}\right|\right)$. This definition gives the duration time about several tens of seconds, and its validity is described a bit later.

Figure 3 shows the relation between the duration time and moment release amount calculated from $M_{\mathrm{o}}=\dot{M}_{\mathrm{o}} \Delta t$ (see Fig. 2(d) for SA), which suggests that slip events in LA and SA belongs to regular earthquake group and very lowfrequency event (VLF) (Ito et al., 2007) of slow earthquake group (Ide et al., 2007), respectively.

On the linear scaling relation between moment and characteristic duration in Fig. 3, the difference in slope between the groups of regular and slow earthquake is thought to be derived from the decay of moment rate proportional to $f^{-1}$ and $f^{-2}$ respectively, where $f$ is frequency (Ide et al., 2007). The $f^{-1}$ decay for the slow earthquake group implies that moment rate $\left(\dot{M}_{\mathrm{o}}\right)$ is constant (Ando et al., 2010), which means that the moment release acceleration $\left(d \dot{M}_{\mathrm{o}} / d t=\ddot{M}_{\mathrm{o}}\right)$ is zero. Figure 2(d) shows a slip event in SA that approximately satisfies the condition of constant moment rate for several tens of seconds, which is verified for most slip events in SAs. Therefore, the definition of the duration time on the basis of lower moment release acceleration is largely consistent with the characteristics of slow earthquake group.

These results indicate that the generation process of short-duration (several tens of seconds) slow earthquake such as VLF swarms may be explained by a chain reac- 

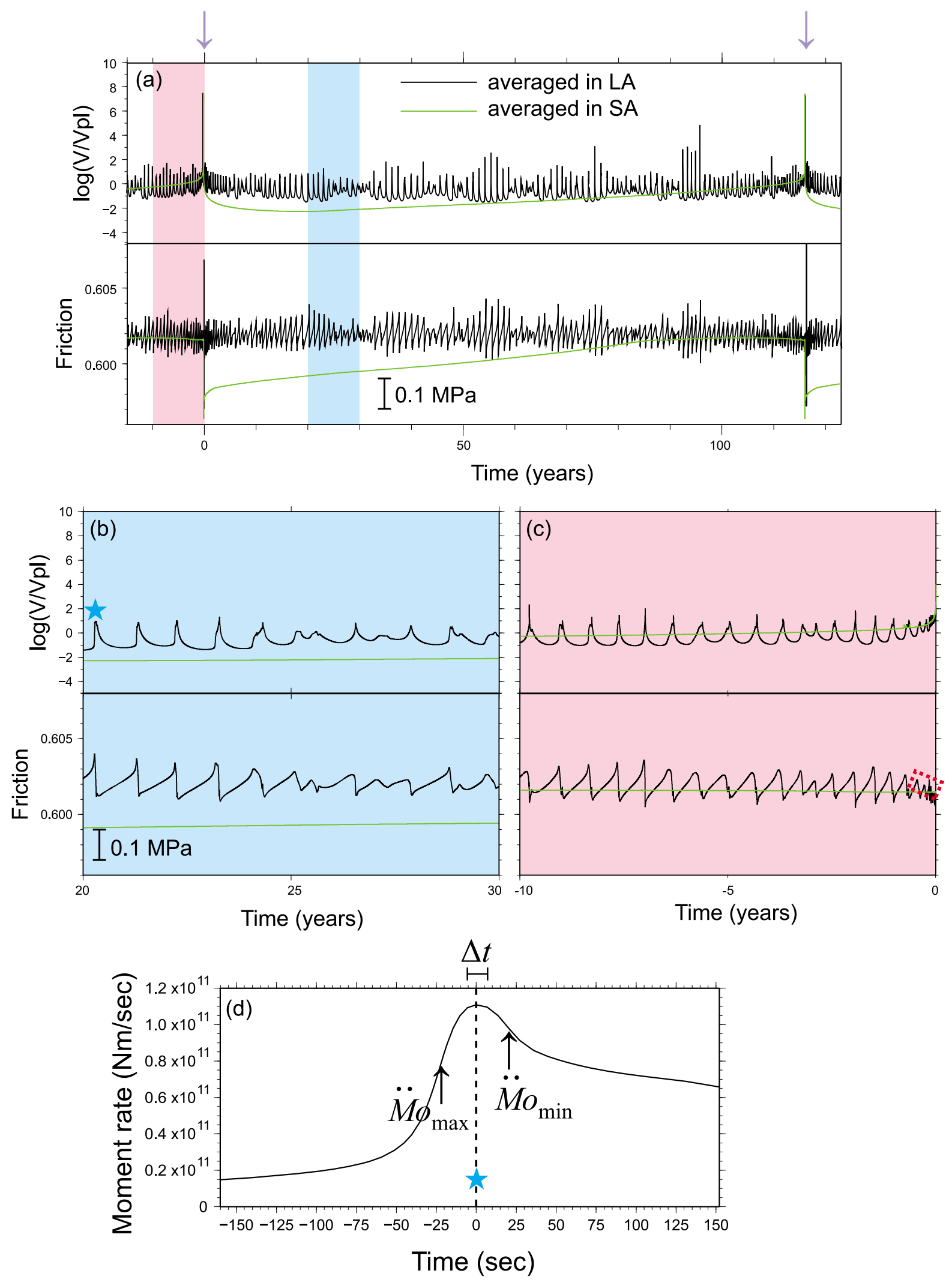

Fig. 2. (a) Time histories of the common logarithm of slip velocities (upper) and friction (lower) averaged in the areas of LA (green) and SA (black) indicated by the arrow in Fig. 1(b). The origin time is set to the occurrence of the megathrust earthquake. The time spans indicated in cyan and magenta correspond to the representative time spans of (b) the interseismic stage and (c) the preseismic stage, respectively. Broken red rectangle in (c) represents slip events with low healing of frictional strength. (d) Time history of the moment release rate for the slip event indicated by star in (b). The origin time is set to the peak of the moment release rate. Maximum and minimum values of moment release acceleration $\left(\ddot{M}_{\text {omax }}\right.$ and $\left.\ddot{M}_{\text {omin }}\right)$ are +2.9 and $-1.0\left[\times 10^{9} \mathrm{~N} \mathrm{~m} / \mathrm{s}^{2}\right]$, respectively. Duration time $(\Delta t)$ of the slow earthquake event is 13 seconds, when it is defined as $\left|\ddot{M}_{\mathrm{o}}(t)\right|<1 / 2\left|\ddot{M}_{\mathrm{omin}}\right|$. 


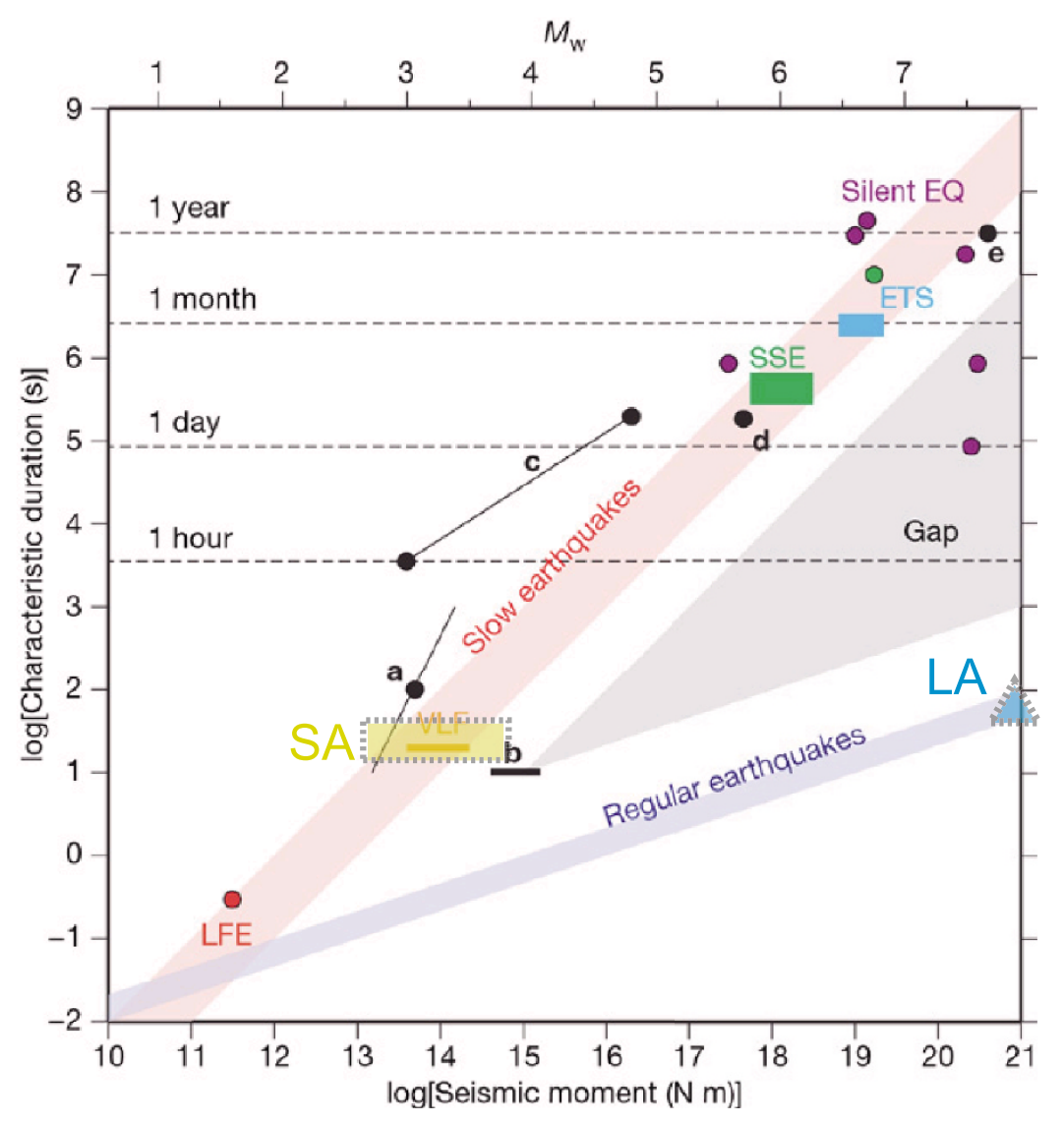

Fig. 3. Relation between duration time and moment release amount for our simulation (symbols enclosed by dotted black curves) of slip events occurring in LA (dark yellow rectangle) and SA (cyan triangle) compared to the empirical scaling law of Ide et al. (2007). The other symbols are observed data.

tion between SAs (Matsuzawa et al., 2004) under high pore pressure. In this study, we classify the slip events in each SA into VLF events according to their slip duration times and seismic moments.

\section{Long-term Change in the Migration Speed of VLF Swarms}

The preseismic slip of the megathrust earthquake could affect the migration speed of VLF swarms as well as their recurrence interval as seen in Fig. 2. Figures 4(a) and 4(b) show the spatiotemporal evolution of the slip velocity normalized by $V_{\mathrm{pl}}$ at $115 \mathrm{~km}$ down-dip from the trench (along green line in Figs. 4(d) and 4(e)) in the interseismic and preseismic stages, respectively. Close-up of the slip velocity pattern in the rectangle in Fig. 4(b) is shown in Fig. 4(c). Figures 4(d) and 4(e) show the snapshots of the normalized slip velocity 20 years after and 0.86 years before a megathrust earthquake, respectively. Based on Figs. 4(ac), we calculate the migration speeds of VLF swarms by tracking transients with slip rate ranging from 2 to $10 V_{\mathrm{pl}}$ (indicated by yellow color). Periods of larger slip rate (from 10 to $100 V_{\mathrm{pl}}$ indicated by orange colors) are difficult to find in Fig. 4(b) because of their short duration, except for times later than -0.2 years in Fig. 4(b).

The dominant migration speed is calculated to be approximately 0.3 to $1 \mathrm{~km}$ /day during the interseismic stage (Fig. 4(a)), while 1 to $3 \mathrm{~km} /$ day in the preseismic stage (Fig. 4(b)). Therefore, the simulation results suggest that monitoring of the migration speeds of VLF swarms as well as recurrence intervals are useful to forecast great earthquakes.

Approximately one month before the megathrust earthquake, Fig. 4(b) shows that the dominant slip velocity for $\mid$ Strike $\mid<40 \mathrm{~km}$ becomes higher than $10 V_{\mathrm{pl}}$ (orange) and is sustained over a long duration time (more than one month). This implies that the moment release rates of VLF swarms near the locked region of LA just before a megathrust earthquake tend to be significantly higher than that in the interseismic stage.

Figure 4(d) suggests that the slip velocity is approximately less than $0.5 V_{\mathrm{pl}}$ (aqua) in the region surrounding the SA (boxed area) and less than $0.1 V_{\mathrm{pl}}$ (blue) dominantly along the center of the SA belt (green line), except for the region where a VLF migration occurs (yellow and orange). Figure 4(e) suggests that the area of higher slip velocity (orange) covering SAs in the preseismic stage tends to be larger than in the interseismic stage as shown in Fig. 4(d), and there is no region in which the slip velocity is less than $0.1 V_{\mathrm{pl}}$. Slip velocity in LA (indicated by green curve in the top of Fig. 2(c)) becomes higher due to the preseismic slip, especially about one year before the megathrust earthquake. These results mean that preseismic slip of LA promotes higher moment release rates of VLF due to its higher slip velocity.

In Fig. 2(c), the stress drops of the slip events become smaller just before a megathrust earthquake, because the re- 


\section{Strike $(\mathrm{km})$}

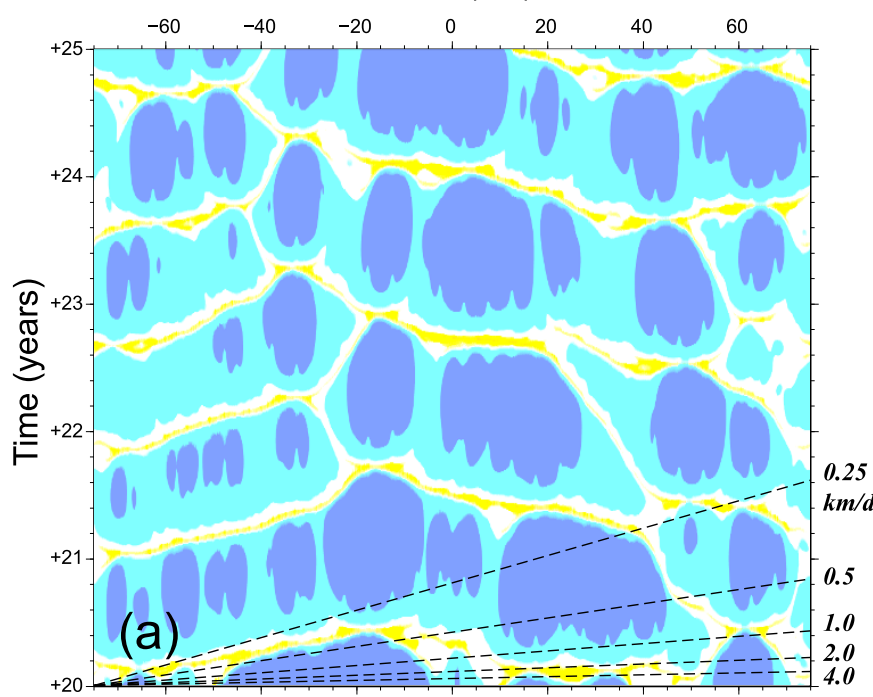

Strike $(\mathrm{km})$

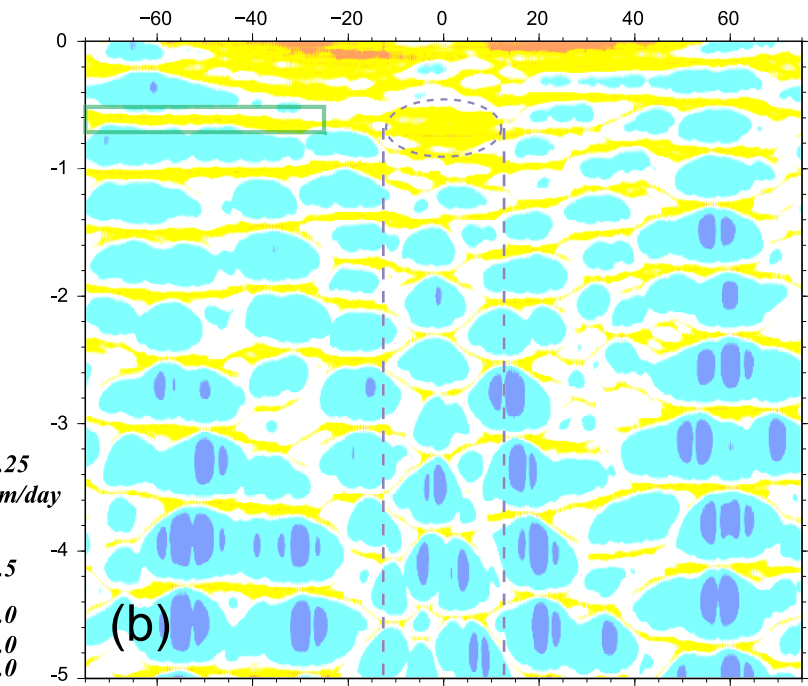

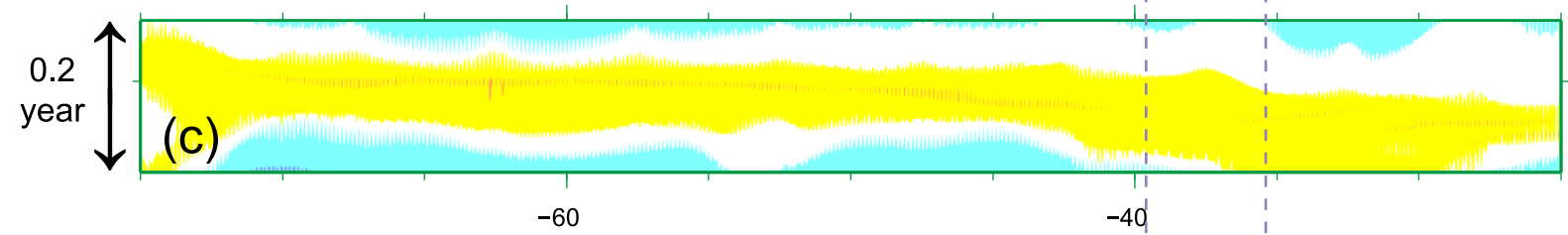

Strike $(\mathrm{km})$
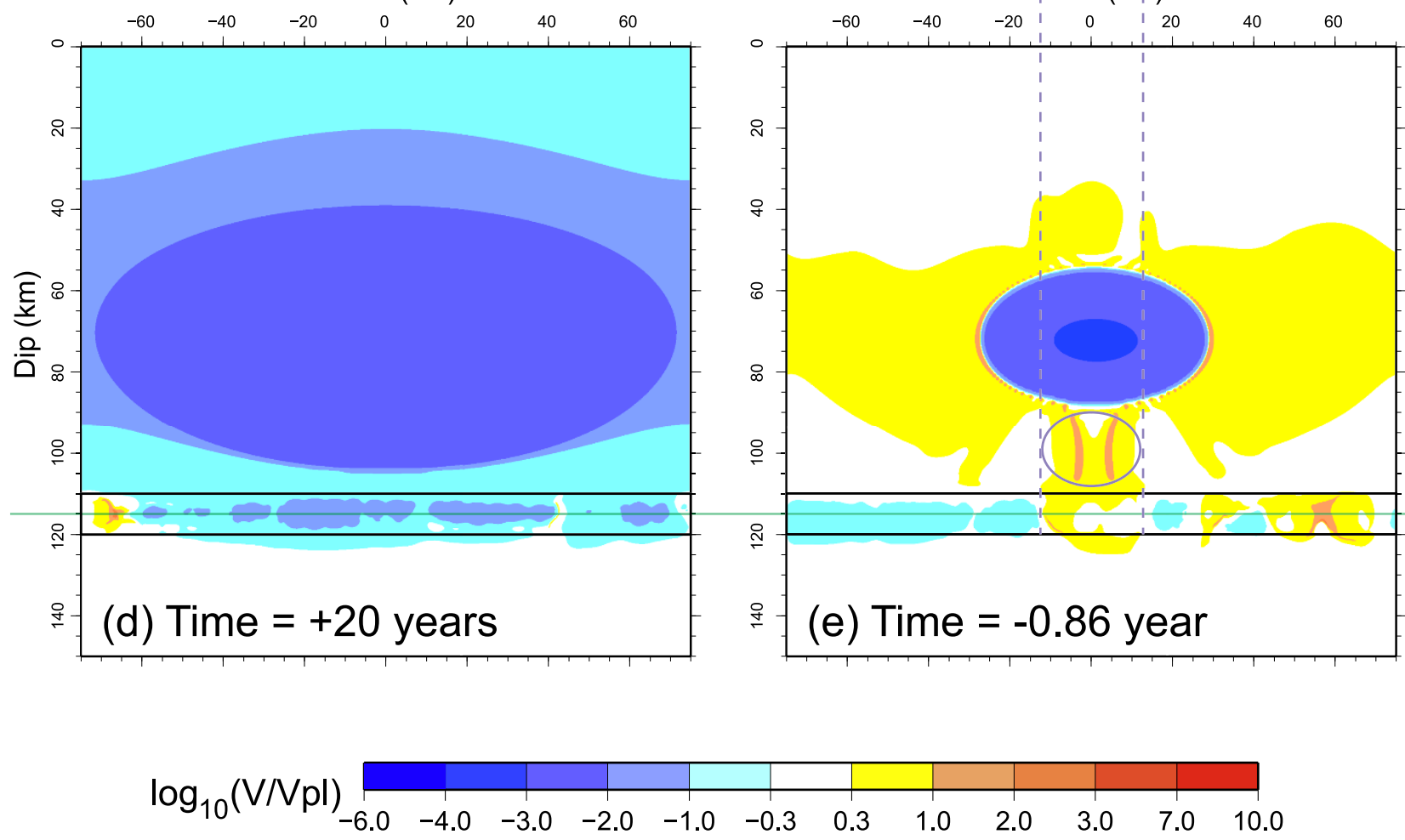

Fig. 4. (a) (b) Spatiotemporal evolution of slip velocities at the "Dip" of $115 \mathrm{~km}$ along strike (green line in Figs. 4(d) and 4(e)) in the interseismic and preseismic stages, respectively. The broken lines in (a) denote the migration speed in $\mathrm{km} / \mathrm{day}$. (c) Close up of the slip velocity evolution in the spatiotemporal region enclosed by the green rectangle in (b), keeping the aspect ratio of space to time. (d) (e) Snapshots of the slip velocity field (d) 20 years after and (e) 0.86 year before the occurrence time of the megathrust earthquake. The ellipse enclosed by the purple curve in (e) represents a large aseismic slip event activating slow earthquakes as shown by the ellipse in (b).

currence interval of SA to heal the frictional strength (represented by the state value $\theta$ ) in Eq. (4)) becomes shorter due to the higher loading rate of shear stress driven by preseismic slip. This lower frictional shear stress makes chain-reaction with higher propagation speed because time required for peeling off each locked region of SA becomes shorter (Ariyoshi et al., 2007b). 
(a)
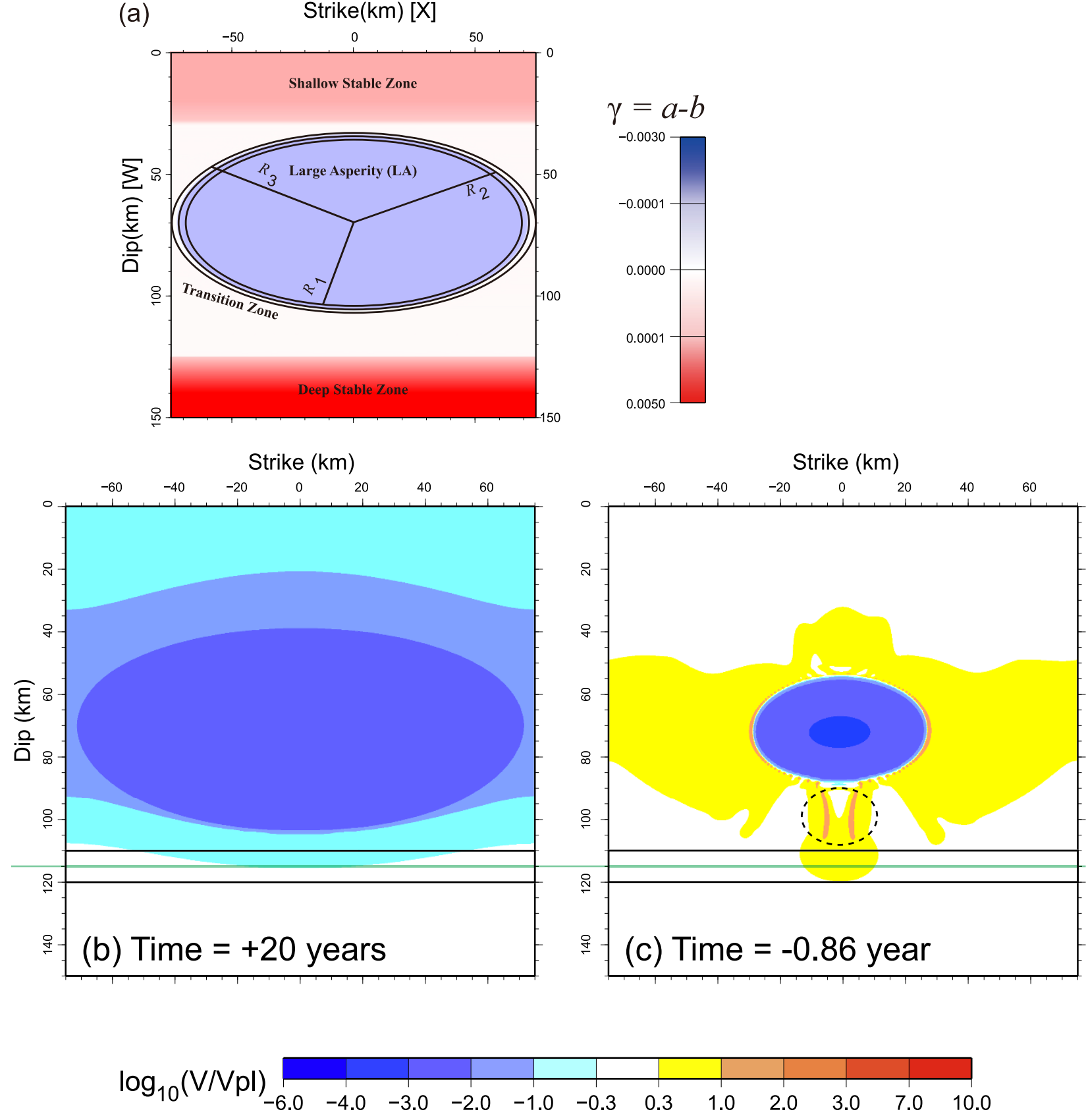

Fig. 5. (a) Spatial distribution of frictional parameter $\gamma=a-b$ for a test model excluded all SAs from original one corresponding to Fig. 1. (b) (c) Snapshots of the slip velocity field for the test model (b) 20 years after and (c) 0.86 year before the occurrence time of the megathrust earthquake, corresponding to Figs. 4 (d) and (e), respectively.

\section{Discussions}

5.1 Effect of numerous small asperities and friction laws on slow earthquake migration

Some recent studies have shown that SSE migration can be reproduced in some models without small asperities (Liu and Rice, 2005; Shibazaki and Shimamoto, 2007), which seems to indicate that the VLF migration might also be reproduced in our model without SAs.

Figure 5(a) shows a test model excluding SAs from the original one corresponding to Fig. 1. In the test model, $T_{\mathrm{r}}=115.7$ years, $M_{\mathrm{w}}=7.9$ and seismic coupling is $74 \%$ for LA. Figures 5(b) and 5(c) show the snapshots of slip velocity field at the same times as Figs. 4(d) and 4(e). The similarity of the slip velocity field around LA between Figs. 4(d-e) and 5(b-c) indicates that the stress perturbations generated by SAs have a negligible effect on LA. As shown in Figs. 5(b) and 5(c), the test model has neither VLF nor SSE migration at any time. This shows that VLF swarms in our first model are not driven by larger scale slow slip transients but result from a chain reaction between SAs, a cascade of triggering through static and creep stresses.

This result seems different from the recent simulation studies reproducing SSE migration (Liu and Rice, 2005; Shibazaki and Shimamoto, 2007). This is probably due to the difference in the physical processes generating the SSE migration. In the previous simulations, SSE migration occurs only in part of the frictionally unstable-stable transition 
zone where a great seismogenic segment is locally peeling off (Liu and Rice, 2005), or in a zone frictionally unstable for slow slip $\left(<10^{-10} \mathrm{~m} / \mathrm{s}\right.$; including SSE) and stable for rapid slip $\left(>10^{-4} \mathrm{~m} / \mathrm{s}\right.$; including seismic slip) (Shibazaki and Shimamoto, 2007).

With respect to the RSF in Eq. (3), Ampuero and Rubin (2008) have recently pointed out that the range of frictional parameters permitting the generation of SSE for the slip law is much less than that for the slowness law in Eq. (4). This means that the slow earthquake migration in the preseismic stage of megathrust earthquakes for the slip law under very low effective normal stress might be different from that for the slowness law.

Just after the occurrence of megathrust earthquakes, seismic slip covers the SA belt (Fig. 2(a)) and the shallow stable zone. Seismic slip in these depth ranges is also observed in the model without SAs. These results indicate that the spatial distribution of seismic slip generated by the megathrust earthquakes extends around the depth of $30 \mathrm{~km}$ if effective normal stress is low there. Therefore, it would be important to robustly estimate the depth range of coseismic slip for previous events such as the 1944 Tonankai earthquake (e.g., Kikuchi et al., 2003) and 1946 Nankai earthquake (e.g., Baba and Cummins, 2005), in order to judge whether high pore pressure exists around the depth of $30 \mathrm{~km}$ or not.

To determine the appropriate friction law and the value of effective normal stress for actual subduction zones, more rock laboratory experiments and in-situ observations such as those by the deep ocean-floor drilling vessel "Chikyu" (e.g., Tobin et al., 2009) are needed. In the following sections, we discuss our simulation results on the basis of the slowness law.

\subsection{Relation of slow earthquake migration with scale factor of frictional instability}

The amount of preseismic slip is strongly dependent on the scale of $d_{\mathrm{c}}$ in the asperity (e.g., Kato et al., 1997). In the present study, we assume $\left(d_{\mathrm{c} 1}, d_{\mathrm{c} 2}\right)=(10,0.43)[\mathrm{mm}]$ for LA and SAs, which is much smaller than in previous studies, e.g., $d_{\mathrm{c}}=200 \mathrm{~mm}$ (Kato et al., 1997) and 60 to $120 \mathrm{~mm}$ (Liu and Rice, 2005). Note that we found no significantly qualitative differences in the simulation results when the cell size was reduced by half under different initial conditions.

Kato (2003) discussed that the characteristics of repeating slip events in an asperity are controlled by the ratio of the asperity radius $\left(r_{\mathrm{a}}\right)$ to the critical (minimum) size of asperity that can generate spontaneous stick-slip instabilities $h^{*}=\eta G d_{\mathrm{c}} / \sigma(b-a)($ Rice, 1993), where $\eta$ is a geometrical factor. His simulation results showed that episodic aseismic slip events including slow earthquakes occur in case of $r_{\mathrm{a}} \sim h^{*}$, and various types of slow earthquakes can be reproduced by changing the value of $r_{\mathrm{a}} / h^{*}$. In this study, the values of $2 r_{2} / h^{*}$ (corresponding to $r / r_{\mathrm{c}}$ in Kato (2003)) for SA are about 1.2-2.0 (Note that Kato (2003) treated $h^{*}$ as a critical radius of asperity while we use it as a critical diameter in this study). These results indicate that we should investigate the migration process of LFT and SSE in addition to VLF, defined by the scaling relation in Fig. 3 by introducing numerous small asperities with different size and/or frictional instability.
When the value of $d_{\mathrm{c} 2}$ was increased by twice so as to reduce the value of $2 r_{2} / h^{*}$ for SA by half as another test model, we found that similar chain-reaction migrations with a tendency to lower $V_{\max }$ and longer duration time $(\Delta t)$ than the original model. Since $\Delta t$ increases drastically with $\left(2 r_{2} / h^{*}\right)^{-1}$ and $M_{\mathrm{o}}$ for single asperity does not fall by ten times with five-times increase in $\left(2 r_{2} / h^{*}\right)^{-1}$ for slower $\left(V<1.0^{-2} \mathrm{~m} / \mathrm{sec}\right)$ slip velocity component (Kato, 2003), these results indicate that characteristics of our simulated VLF migration process may be applicable to SSE by integration of SAs in accordance with its migration. Since LFTs occur in a smaller area (Ito et al., 2007) with shorter duration time and moment release rate comparable to VLF (Ide et al., 2007), LFT is thought to have higher slip velocity than VLF. This means that LFT migration may also be reproduced by numerous asperities of smaller size and frictionally more instability with greater $r_{\mathrm{a}} / h^{*}\left(2 r_{2} / h^{*}\right)$ due to shorter $d_{\mathrm{c}}$, if the value of $\gamma$ is common to VLF and LFT because of the same temperature and depth (e.g., Blanpied et al., 1998). Since the models with greater number of asperities having shorter time scale than the original model in Fig. 1 need heavier computations, simulations of LFT migration are left for a future study.

To discuss the range of frictional parameters adequate for VLF, we compare the migration speed of VLF between simulated and observed results quantitatively. From previous simulation studies, the migration speed of post-seismic slip is inversely proportional to $d_{\mathrm{c}}$ (Ariyoshi, 2006). If this relation is applicable to VLF, the adequate value of $d_{\mathrm{c}}$ for VLF could be roughly estimated by $\left[d_{\mathrm{c} 2} \times(\right.$ simulated $\div$ observed migration speed; $\mathrm{km} /$ day $)=\left(0.43 \times 10^{-3}\right) \times(1 \sim 3 / 2 \sim$ $\left.20) \fallingdotseq 0.6 \sim 0.02 \times 10^{-3} \mathrm{~m}\right]$. On the other hand, lower effective normal stress causes drastically faster migration of postseismic slip (Ariyoshi et al., 2007a). Since the application of $\kappa=0.1$ in this study as described in Section 2 is a rough estimation (Wang and Suyehiro, 1999; Ariyoshi et al., 2007a), a little lower effective normal stress may also explain the migration speed quantitatively.

In the following section, we discuss the qualitative similarity between our simulation results and observed slow earthquake migrations including LFT and SSE.

\subsection{Similarity between the simulated slow earthquake migration and observational results}

Figure 4(e) shows that a large aseismic slip event occurs locally between LA and the SA belt (indicated by the ellipse) approximately one year before the megathrust earthquake. Since this event is common to the model without the SA belt (Fig. 5(c)), it is not triggered by the slow earthquake migration in the SA belt. On the other hand, the spatiotemporal region enclosed by the ellipse in Fig. 4(b) shows that the large aseismic slip event triggers slow earthquakes in the SA belt with a shorter recurrence interval compared to other areas ( $\mid$ Strike $\mid>20 \mathrm{~km})$. The migration distance in the SA belt corresponds to the size of the large aseismic slip region ( $\mid$ Strike $\mid<20 \mathrm{~km}$ ). This behavior is similar to the long-term SSE observed at Bungo Channel in 2003, where nearby LFT migration had occurred either at a shorter recurrence interval or nearly continuously for several months (Obara, 2008). Therefore, the activity of the LFTs may be useful to estimate the duration and the location of lo- 
cal aseismic slip events, such as the long-term SSE and the preseismic slip in the deeper part of LA.

As pre- and post-seismic changes, intense LFT activity began to occur almost directly below the 2004 Parkfield earthquake about three weeks before the earthquake and has continued only apart from the hypocenter over for four years (Nadeau and Guilhem, 2009; Shelly, 2009), which means that the distance from the hypocenter of triggering earthquake may also affect the sensitivity of LFT to preand post-seismic slip (Shelly, 2009).

Our simulation shows that the moment release rate of VLF events near the locked region of LA becomes higher about one month before the megathrust earthquake as mentioned in Section 3 (Fig. 4(b)), and Fig. 2(a) shows that a shorter recurrence interval of VLF events in the SA belt occurs several years after the megathrust earthquake. These simulation results are consistent with those results observed in Parkfield for the pre- and post-seismic stages of nearby large earthquakes.

Since SSE, pre- and post-seismic slips are all transients of interplate-slip faster than $V_{\mathrm{pl}}$, the observations reported by Obara (2008), Nadeau and Guilhem (2009), and Shelly (2009) would support our suggestion that the preseismic slip of megathrust earthquakes can be practically detected by monitoring slow earthquake migrations, even if the actual $d_{\mathrm{c}}$ is less than several centimeters.

\section{Conclusions}

The simulation results of the present study, assuming slowness law of RSF, show that swarms of slip events occurring in numerous small asperities under low effective normal stress (about 40-50 MPa) condition and close-set geometry can explain the lower rate of moment release as observed for slow earthquakes (Ide et al., 2007). This result may explain the observed migration process of VLF swarms (Obara and Sekine, 2009).

The simulation results also suggest that the activity of VLF swarms in the preseismic stage of great interplate earthquakes tends to show higher migration speed and higher moment release rate as well as shorter recurrence interval.

Since slow earthquake migration has only recently been observed, we have not yet observed their migration process just before megathrust earthquakes in subduction zones. In order to succeed in detecting their precursory changes robustly, it is important to monitor the change in slow earthquake activity in addition to crustal deformations and the activity change of regular earthquakes.

Acknowledgments. The authors would like to thank Dr. Kazushige Obara and Dr. Bunichiro Shibazaki for their fruitful discussion, which helped to narrow the target of our simulation. Thoughtful comments from N. Kato and an anonymous reviewer kindly helped us to improve this manuscript. The present study used the Earth Simulator and the supercomputing resources at the Cyberscience Center of Tohoku University. GMT software (Wessel and Smith, 1998) was used to draw a number of the figures. The present study was supported by the DONET program of the Ministry of Education, Culture, Sports, Science and Technology.

\section{References}

Ampuero, J.-P. and A. M. Rubin, Earthquake nucleation on rate and state faults: Aging and slip laws, J. Geophys. Res., 113, B01302, doi:10. 1029/2007JB005082, 2008.

Ando, R., R. Nakata, and T. Hori, A slip pulse model with fault heterogeneity for low-frequency earthquakes and tremor along plate interfaces, Geophys. Res. Lett., 37, L10310, doi:10.1029/2010GL043056, 2010.

Ariyoshi, K., Study on the generation process of interplate earthquakes based on numerical simulations, Doctor Thesis of Tohoku Univ., Japan, 136 pp., 2006 (in Japanese).

Ariyoshi, K., T. Matsuzawa, and A. Hasegawa, The key frictional parameters controlling spatial variations in the speed of postseismic-slip propagation on a subduction plate boundary, Earth Planet. Sci. Lett., 256, 136-146, doi:10.1016/j.epsl.2007.01.019, 2007a.

Ariyoshi, K., T. Matsuzawa, R. Hino, and A. Hasegawa, Triggered nonsimilar slip events on repeating earthquake asperities: Results from 3D numerical simulations based on a friction law, Geophys. Res. Lett., 34, L02308, doi:10.1029/2006GL028323, 2007b.

Baba, T. and P. R. Cummins, Contiguous rupture areas of two Nankai Trough earthquakes revealed by high-resolution tsunami waveform inversion, Geophys. Res. Lett., 32, L08305, doi:10.1029/2004GL022320, 2005.

Blanpied, M. L., C. J. Marone, D. A. Lockner, J. D. Byerlee, and D. P. King, Quantitative measure of the variation in fault rheology due to fluid-rock interactions, J. Geophys. Res., 103, 9691-9712, 1998.

Boatwright, J. and M. Cocco, Frictional constraints on crustal faulting, $J$. Geophys. Res., 101, 13895-13909, 1996.

Dieterich, J. H., Modeling of rock friction: 1. Experimental results and constitutive equations, J. Geophys. Res., 84, 2161-2168, 1979.

Dragert, H., Mediating plate convergence, Science, 315, 471-472, doi: 10.1126/science.1137171, 2007.

Hacker, B. R., S. M. Peacock, G. A. Abers, and S. D. Holloway, Subduction factory 2. Are intermediate-depth earthquakes in subducting slabs linked to metamorphic dehydration reactions?, J. Geophys. Res., 108(B1), 2030, doi:10.1029/2001JB001129, 2003.

Ide, S., G. C. Beroza, D. R. Shelly, and T. Uchide, A new scaling law for slow earthquakes, Nature, 447, 76-79, doi:10.1038/nature05780, 2007.

Ito, Y., K. Obara, K. Shiomi, S. Sekine, and H. Hirose, Slow earthquakes coincident with episodic tremors and slow slip events, Science, 315, 503-506, 2007.

Kato, N., Repeating slip events at a circular asperity: Numerical simulation with a rate- and state-dependent friction law, Bull. Earthq. Res. Inst. Univ. Tokyo, 78, 151-166, 2003.

Kato, N., M. Ohtake, and T. Hirasawa, Possible mechanism of precursory seismic quiescence: Regional stress relaxation due to preseismic sliding, Pure Appl. Geophys., 150, 249-267, 1997.

Kikuchi, M., M. Nakamura, and K. Yoshikawa, Source rupture processes of the 1944 Tonankai earthquake and the 1945 Mikawa earthquake derived from low-gain seismograms, Earth Planets Space, 55, 159-172, 2003.

Liu, Y. and J. R. Rice, Aseismic slip transients emerge spontaneously in three-dimensional rate and state modeling of subduction earthquake sequences, J. Geophys. Res., 110, B08307, doi:10.1029/2004JB003424, 2005.

Matsubara, M., K. Obara, and K. Kasahara, Three-dimensional P- and Swave velocity structures beneath the Japan Islands obtained by highdensity seismic stations by seismic tomography, Tectonophysics, $\mathbf{4 5 4}$, 86-103, 2008.

Matsuzawa, T., N. Uchida, T. Igarashi, T. Okada, and A. Hasegawa, Repeating earthquakes and quasi-static slip on the plate boundary east off northern Honshu, Japan, Earth Planets Space, 56, 803-811, 2004.

Miyazaki, S., P. Segall, J. J. McGuire, T. Kato, and Y. Hatanaka, Spatial and temporal evolution of stress and slip rate during the 2000 Tokai slow earthquake, J. Geophys. Res., 111, B03409, doi:10. 1029/2004JB003426, 2006.

Nadeau, R. M. and A. Guilhem, Nonvolcanic tremor evolution and the San Simeon and Parkfield, Califorinia, Earthquakes, Science, 325, 191-193, doi:10.1126/science.1174155, 2009.

Nakata, R., N. Suda, and H. Tsuruoka, Non-volcanic tremor resulting from the combined effect of Earth tides and slow slip events, Nature Geosci., 1, 676-678, doi:10.1038/ngeo288, 2008.

Obara, K., Nonvolcanic deep tremor associated with subduction in southwest Japan, Science, 296, 1679-1681, doi:10.1126/science.1070378, 2002.

Obara, K., Phenomenology of deep slow earthquake family in southwest 
Japan-Spatiotemporal characteristics and segmentation-, Eos Trans. $A G U, \mathbf{8 9}(52)$, Fall Meet. Abstract U33A-0035, 2008.

Obara, K. and S. Sekine, Characteristic activity and migration of episodic tremor and slow-slip events in central Japan, Earth Planets Space, 61, 853-862, 2009.

Okada, Y., Internal deformation due to shear and tensile faults in a halfspace, Bull. Seismol. Soc. Am., 82, 1018-1040, 1992.

Rice, J. R., Spatio-temporal complexity of slip on a fault, J. Geophys. Res., 98, 9885-9907, 1993.

Rogers, G. and H. Dragert, Episodic tremor and slip on the Cascadia subduction zone: The chatter of silent slip, Science, 300, 1942-1943, 2003.

Rubin, A. M. and J.-P. Ampuero, Earthquake nucleation on (aging) rate and state faults, J. Geophys. Res., 110, B11312, doi:10. 1029/2005JB003686, 2005.

Rubinstein, J. L., M. L. Rocca, J. E. Vidale, K. C. Creager, and A. G. Wech, Tidal modulation of nonvolcanic tremor, Science, 319, 186-189, doi:10.1126/science.1150558, 2008.

Ruina, A., Slip instability and state variable friction laws, J. Geophys. Res., 88, 10,359-10,370, 1983.

Savage, J. C., A dislocation model of strain accumulation and release at a subduction zone, J. Geophys. Res., 88, 4984-4996, 1983.

Schwartz, S. Y. and J. M. Rokosky, Slow slip events and seismic tremor at circum-Pacific subduction zones, Rev. Geophys., 45, RC3004,
doi:10.1029/2006RG000208, 2007.

Shelly, D. R., Possible deep fault slip preceding the 2004 Parkfield earthquake, inferred from detailed observations of tectonic tremor, Geophys. Res. Lett., 36, L17318, doi:10.1029/2009GL039589, 2009.

Shelly, D. R., G. C. Beroza, S. Ide, and S. Nakamura, Low frequency earthquakes in Shikoku, Japan, and their relationship to episodic tremor and slip, Nature, 442, 188-191, doi:10.1038/nature04931, 2006.

Shibazaki, B. and T. Shimamoto, Modeling of short-interval silent slip events in deeper subduction interfaces considering the frictional properties at the unstable-stable transition, Geophys. J. Int., 171, 191-205, doi:10.1111/j.1365-246X.2007.03434.x, 2007.

Tobin, H. et al., NantroSEIZE Stage 1 Expeditions 314, 315, and 316: First Drilling Program of the Nankai Trough Seismogenic Zone Experiment, Scientific Drilling, 8, 4-17, doi:10.2204/iodp.sd.8.01.2009, 2009.

Wang, K. and K. Suyehiro, How does plate coupling affect crustal stresses in northeast and southwest Japan, Geophys. Res. Lett., 26, 2307-2310, doi:10.1029/1999GL900528, 1999.

Wessel, P. and W. H. F. Smith, New, improved version of the Generic Mapping Tools released, Eos Trans. AGU, 79, 579, 1998.

K. Ariyoshi (e-mail: ariyoshi@jamstec.go.jp), T. Matsuzawa, J.-P. Ampuero, R. Nakata, T. Hori, Y. Kaneda, R. Hino, and A. Hasegawa 